\title{
Bi-Level Optimization as a Tool for Implementation of Intelligent Transportation Systems
}

\author{
Krasimira Stoilova, Todor Stoilov, Vladimir Ivanov \\ Institute of Information and Communication Technologies, Bulgarian Academy of Sciences, \\ 1113 Sofia, Bulgaria \\ E-mails: k.stoilova@hsi.iccs.bas.bg todor@hsi.iccs.bas.bgｉvanov.vladi@gmail.com
}

\begin{abstract}
The Intelligent Transportation System (ITS) is used as a term for integrating requirements and functionalities towards transportation systems, which in urban environment raises complex exploitation and control problems. Important part of the ITS is the control which has to be applied for traffic flows. The control processes are strongly linked with requirements and targets for optimization of the transportation behavior. The paper applies new optimization formal description of control by bi-level optimization. Except the trivial traffic lights control, the bi-level formalization allows additional traffic characteristics to be defined like maximal/minimal values. The paper defines, solves and provides numerical simulations for minimization of the vehicle queues in front of the traffic lights. Such bi-level optimization problem is applied simultaneously for maximization the traffic flows on arterial and important directions of the urban transportation network. The formal description of the bi-level problem is provided. The results of the bi-level control have been compared with the cases of single optimization of the vehicles queues. The simulation results prove that the bi-level problem gives benefits satisfying an additional goal, which improves additional characteristic of the transport behavior. The bi-level optimization formalism can be used as a tool for implementation of integration of ITS control policies.
\end{abstract}

Keywords: Bi-level optimization, hierarchical multilevel systems, transportation, traffic control.

\section{The paradigm of intelligent transportation systems}

The Information Technologies (IT) have impact on the domain of transportation systems. Different components of the transportation systems become intelligent by inclusion of microcontrollers and software components implementing new additional functionalities to vehicles, message signs, traffic lights. The main goal of the Intelligent Transportation System (ITS) is to reduce the congestions on roads, to 
assure softy and efficiency of the transportation services, to provide information services to the travelers [1].

ITS are regarded as a new technology field which integrates activities and services such as transport management, control of transportation systems, control methods, infrastructural design [17]. ITS comprise services for highway control, implementation of navigation systems, integration and freight control in air, water and rail transport. Following [17], four generations of ITS are estimated: ITS 1.0 before 2000 - One-way infrastructure based; ITS2.0, 2000-2003 - Two-ways communication technology; ITS3.0, 2004-2005 - Automated vehicle operations and system management; ITS4.0, 2006-2011 - Multi-modal transport, information networks for system operations.

Now, the connected vehicle control is assumed as important task which is worked out by methodologies applied in ITS [10]. The domains where ITS have wide penetrations are [17]:

- Arterial and freeway management systems;

- Freight management systems;

- Transit management systems;

- Incident management systems;

- Emerge management systems;

- Regional multimodal and traveler information systems.

This paper is focused on problems related with freeway management systems. The traffic jams are disaster problem encountered in urban regions. Both infrastructural changes and intelligent control are tools to beat the congestion events. The traffic signal control is in primary use for managing the traffic flows in urban area.

The traffic signal control can address many objectives: improvement of traffic flows, safety, coordinate control of traffic signals, adjusting the durations of signal phases, provision of adaptive signal control. The optimization of the traffic signal duration is the general control approach for reduction of congestions. The aim of the traffic signal control is to keep the transportation network on its nominal capacity level. This capacity can be decreased by the congestion for the case of lack of traffic control. For the urban areas the basic goal applied for the traffic management is the minimization of lost time arising in front of the junctions during the red and/or amber lights. The lost time is proportional to the queue lengths arising in the transport network. Thus, the traffic lost time and/or vehicle queues are formalized as optimization criteria in the optimization control problems.

\section{Traffic control system and its optimal operation}

The paper addresses the case of traffic control system in urban network. The control influences which are under optimization calculations concern the green lights on the junctions, the duration of the traffic light cycle $c$ and the offset between the green lights of neighbor junctions. Because the transportation network operates in dynamically changes of the traffic behavior, adaptation of the control influences is needed. An advanced concept for the management of such complex transportation 
system is raised by the paradigm of "autonomic systems" $[10,13,20]$. The term "autonomic" is a biological metaphor adopted by IBM to describe the desired properties of the complex information systems. The implementation of autonomic operations in transportation systems will provide self-management of the transportation control system, according to the changing solutions in urban networks. An application to the autonomic managements is the self-optimization capability of the control system. The self-optimization implies implementation of set of goals for the control process tackling sets of constraints and requirements.

An approach for the application of autonomic principles of control is the usage of new formal description of the control problems, described as bi-level optimization $[18,20]$.

\section{Bi-level models in transportation control}

The bi-level optimization approach currently is regarded as new formal tool to define and solve practical optimization problems. The bi-level optimization problems can be seen in $[6,21]$. Applications of bi-level formalism for real problem are given in [3]. Some practical problems from the transportation domain by bi-level approach are given below.

In $[4,7,16]$ parameters of the traffic flows and traffic planning are optimized in bi-level form. In $[2,5,8,14,15]$ toll pricing model is evaluated to increase the income from toll taxes but respecting the driver preferences for cheap transport. In [11, 12] routing algorithms for hazardous materials are defined by bi-level optimization. Optimal store and routing problem is defined in [9]. These applications belong to problems related with off-line activities: planning, off-line decision making, parameter estimation, pricing off-line routing decisions. Currently, attempts are done for on-line application of bi-level optimization in the control process. In [18] the queue lengths in urban network are minimized and maximal outflow from the network is achieved. In [20] maximization of the arterial traffic flow is targeted and optimized. In [19] the duration of the traffic lights is evaluated by minimization of the queues and going preference for particular outgoing traffic. This paper makes an illustration for the case that the upper level control problem of bi-level optimization can be defined in different manner. For the current case minimization of the waiting time for priority direction is considered simultaneously with the global minimization of the queue lengths of a crossroad section. The calculations can be performed online, achieving intelligent transportation behavior - self-optimization property of the control system.

\section{Case study}

The crossroad section under consideration is an important spot in Sofia. The notations, used in the formal relations, follow these of Fig. 1. The notations $z_{i 0}$, $i=1, \ldots, 8$, are the values of the waiting vehicles/queues in front of the different directions of the junction. The values $u_{1}, u_{2}, u_{3}, u_{4}$ are the durations of the green lights 
of the four phases of the traffic lights. The couple $\left(z_{i 0}, u_{j}\right), i=1, \ldots, 8, j=1, \ldots, 4$, means that the queue $z_{i 0}$ has the right of driving during the green phase $u_{j}$.

Following the infrastructure of the junction in Fig. 1, the store and forward modeling gives a set of relations -

$$
\begin{gathered}
z_{1}=z_{10}+z_{1 i n}-u_{1} s, \\
z_{2}=z_{20}+z_{2 i n}-u_{2} s_{z}, \\
z_{3}=z_{30}+z_{3 i n}-\left(u_{2}+u_{3}+u_{4}\right) s_{z}, \\
z_{4}=z_{40}+z_{4 i n}-u_{3} s_{z}, \\
z_{5}=z_{50}+z_{5 i n}-u_{1} s \\
z_{6}=z_{60}+z_{6 i n}-u_{4} s_{z}, \\
z_{7}=z_{70}+z_{7 i n}-\left(u_{3}+u_{4}\right) s_{z}, \\
z_{8}=z_{80}+z_{8 i n}-\left(u_{1}+u_{2}\right) s_{z},
\end{gathered}
$$

where

$z_{i}, i=1, \ldots, 8$, mean the current length of vehicles for direction $z_{i}$ (vehicles);

$z_{i 0}, i=1, \ldots, 8$, are waiting vehicles (vehicles);

$z_{\text {in }}, i=1, \ldots, 8$, are new coming vehicles for direction $i$ (vehicles);

$u_{j}, j=1,4$, are duration of the green phase of the traffic lights with four phases

(s);

$s$ is capacity for the saturation flow in right direction (vehicles per $1 \mathrm{~s}$ );

$s_{z}$ is capacity for the saturation flow in turning direction (vehicles per $1 \mathrm{~s}$ ).

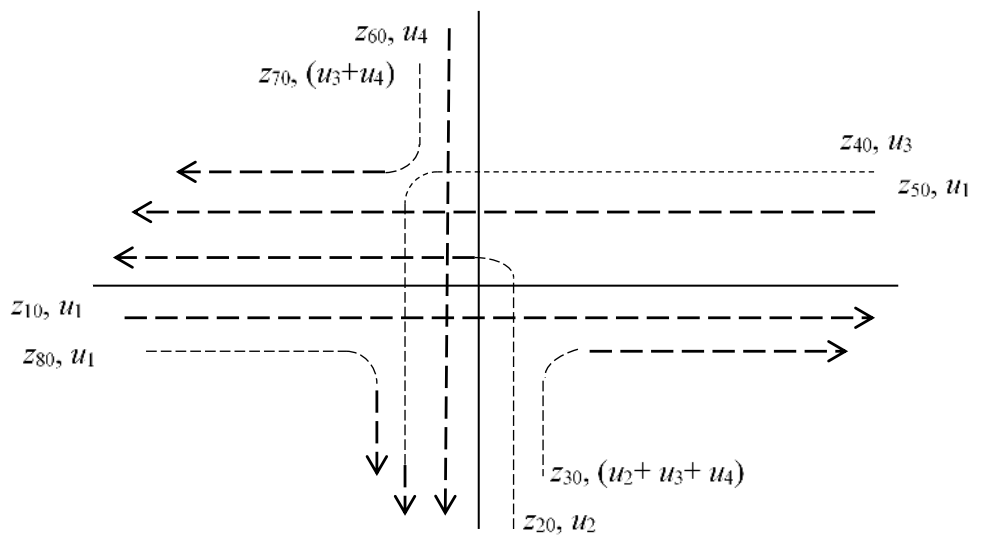

Fig. 1. Crossroad section's architecture

The optimization problem, defined for the bi-level optimization has the form

subject to (1) plus the following relations:

$$
\min \sum_{i=1}^{8} z_{i 0}^{2}
$$

$$
\sum_{j=1}^{4} u_{i}=c, u_{i 1} \leq u_{i} \leq u_{i \mathrm{~h}}, i=1, \ldots, 4,
$$

where $c$ is the duration of the traffic cycle, $u_{i 1}$ (low) and $u_{i \mathrm{~h}}$ (high) are practical requirements for minimal and maximal durations of the green light for phase $i$.

By substitution of (1) in the goal function (2) the optimization problem becomes analytically defined quadratic programming one with arguments $u_{i}, i=1, \ldots, 4$, 
where

$$
\begin{gathered}
\min _{u_{i,}, i=1, \ldots, 4}\left\{u^{\mathrm{T}} Q u+R^{\mathrm{T}} u\right\} \\
\sum_{i=1}^{4} u_{i}=c \\
u_{i 1} \leq u_{i} \leq u_{i \mathrm{~h}}, i=1, \ldots, 4,
\end{gathered}
$$

$$
\begin{gathered}
Q=\left|\begin{array}{cccc}
2 s^{2}+s_{z}^{2} & s_{z}^{2} & 0 & 0 \\
s_{z}^{2} & 3 s_{z}^{2} & s_{z}^{2} & s_{z}^{2} \\
0 & s_{z}^{2} & 3 s_{z}^{2} & 2 s_{z}^{2} \\
0 & s_{z}^{2} & 2 s_{z}^{2} & 2 s_{z}^{2}+s^{2}
\end{array}\right|, \\
R=\left|\begin{array}{c}
-2 z_{10} s-2 z_{50} s-2 z_{80} s_{z} \\
-2 z_{20} s_{z}-2 z_{30} s_{z}-2 z_{80} s_{z} \\
-2 z_{30} s_{z}-2 z_{40} s_{z}-2 z_{70} s_{z} \\
-2 z_{30} s_{z}-2 z_{60} s_{z}-2 z_{70} s_{z}
\end{array}\right| .
\end{gathered}
$$

The upper level optimization problem is defined to give priority for flows $z_{2}$ and z. The priority is defined by decreasing the waiting time for these queues. For the flow $z_{2}$, following Fig. 1, the waiting time is (in s)

(4a)

The same case is for $z_{4}$ :

$$
w_{2}\left(c, u_{2}(c)\right)=c-u_{2} \text {. }
$$

$$
w_{4}\left(c, u_{3}(c)\right)=c-u_{3} .
$$

The upper level optimization problem is defined for minimization of $w_{2}(c), w_{4}(c)$ by changing the duration of the cycle $c$. The formal description of this upper level optimization goal is defined in a quadratic form

$$
\begin{gathered}
\min _{c}\left[w_{2}^{2}(c)+w_{4}^{2}(c)\right], \\
c_{\min } \leq c \leq c_{\max },
\end{gathered}
$$

where the bounds $c_{\min }$ and $c_{\max }$ are defined by practical considerations. The bi-level optimization problem graphically is presented on Fig. 2.

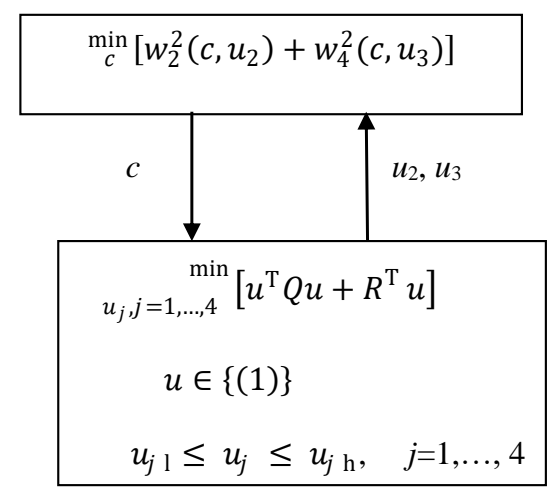

Fig. 2. Bi-level optimization problems

\section{Numerical simulation results}

The bi-level optimization problem from Fig. 2 was solved applying a MATLAB function "solvebilevel". The initial used values are 


$$
\begin{aligned}
& z_{0}^{\mathrm{T}}=\left|\begin{array}{llllllll}
70 & 60 & 70 & 60 & 70 & 60 & 70 & 60
\end{array}\right|, \\
& z_{\text {in }}^{\mathrm{T}}=\left|\begin{array}{llllllll}
0.1 & 0.1 & 0.1 & 0.1 & 0.1 & 0.1 & 0.1 & 0.1
\end{array}\right| \text {, } \\
& s=1 \text { vehicles per } 1 \mathrm{~s}, s_{z}=0.25 \text { vehicles per } 1 \mathrm{~s} \text {. }
\end{aligned}
$$

The control values for $u_{j}, j=1, \ldots, 4$, and $c$ were evaluated in a sequence:

Step 1. Given initial values $z_{i 0}, i=1, \ldots, 8$.

Step 2. Solution of bi-level problem, Fig. 2. The optimal solutions of $u_{j}^{*}$, $j=1, \ldots, 4, c^{*}$ are found.

Step 3. Actualization of the queue lengths $z_{i 0}^{*}, i=1, \ldots, 8$, following Step 1 .

Step 4. Evaluation of the total waiting vehicles and the total waiting time (in s)

$$
\begin{gathered}
x_{\text {total (bi-level) }}=\sum_{i=1}^{8} z_{i 0}^{*}, \\
w_{\text {wait (bi-level) }}=w_{2}+w_{3} .
\end{gathered}
$$

Step 5. Return to Step 1 with the new waiting queues $z_{i 0}^{*}, i=1, \ldots, 8$.

The calculations are provided for several traffic cycles.

These results have been compared with a single optimization policy which applies minimization of the total waiting vehicles $x_{\text {total (single). The corresponding }}$ waiting time $w_{\text {wait (single) }}$ is also calculated. The evaluation for the single optimization is performed by the same bi-level algorithm. On Step 2 the solution of problem (2) is performed by single optimization instead of the bi-level problem on Fig. 2.

Two comparisons have been performed. The single optimization has been

\begin{tabular}{|c|}
\hline Bi-level optimization \\
\hline 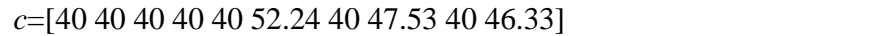 \\
\hline time $=\left[\begin{array}{llllllllll}40 & 80 & 120 & 160 & 200 & 252.24 & 292.24 & 340.77 & 380.77 & 427.1\end{array}\right]$ \\
\hline$X_{\text {total }}=\left[\begin{array}{lllllllllll}474 & 433 & 393 & 357 & 324 & 283 & 251 & 219 & 201 & 179\end{array}\right]$ \\
\hline$w_{\text {wait }}=\left[\begin{array}{llllllllll}123 & 126 & 129 & 127 & 125 & 119 & 116 & 110 & 106 & 100\end{array}\right]$ \\
\hline Single optimization, $c=40$, const \\
\hline time $_{40}=\left[\begin{array}{llllllllll}40 & 80 & 120 & 160 & 200 & 240 & 280 & 320 & 360 & 400\end{array}\right] ;$ \\
\hline 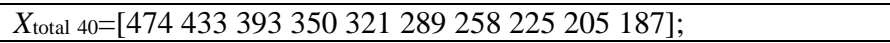 \\
\hline 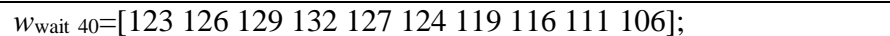 \\
\hline Single optimization, $c=120$, const \\
\hline time $_{120}=\left[\begin{array}{lllll}120 & 240 & 360 & 480\end{array}\right]$ \\
\hline$X_{\text {total } 120}=\left[\begin{array}{lllll}394 & 280 & 205 & 141\end{array}\right]$ \\
\hline$w_{\text {wait } 120}=\left[\begin{array}{lllll}130 & 128 & 118 & 104\end{array}\right]$; \\
\hline
\end{tabular}
applied twice with two different values of the cycle: $c=40 \mathrm{~s}$ and $c=120 \mathrm{~s}$. These values correspond to the upper and lower values of the constraint in the bi-level optimization problem.

The numerical results are given in Table 1 .

The simulation was performed for a time period of $500 \mathrm{~s}$. The bi-level optimization applies variable duration of the traffic light cycle. It is found as optimal solution of the bi-level problem. The single optimization problem applies constant values for the time cycle $c=40 \mathrm{~s}$ and $c=120 \mathrm{~s}$. The graphical comparisons of the numerical results are given in Fig. 3 and Fig. 4. The solutions of the bi-level problem are denoted by solid line on Figs 3 and 4 . The single optimization with duration of the traffic light $c=40 \mathrm{~s}$ is denoted by $(*)$ and the single optimization with duration of the traffic light $c=120 \mathrm{~s}$ is denoted by (+). 


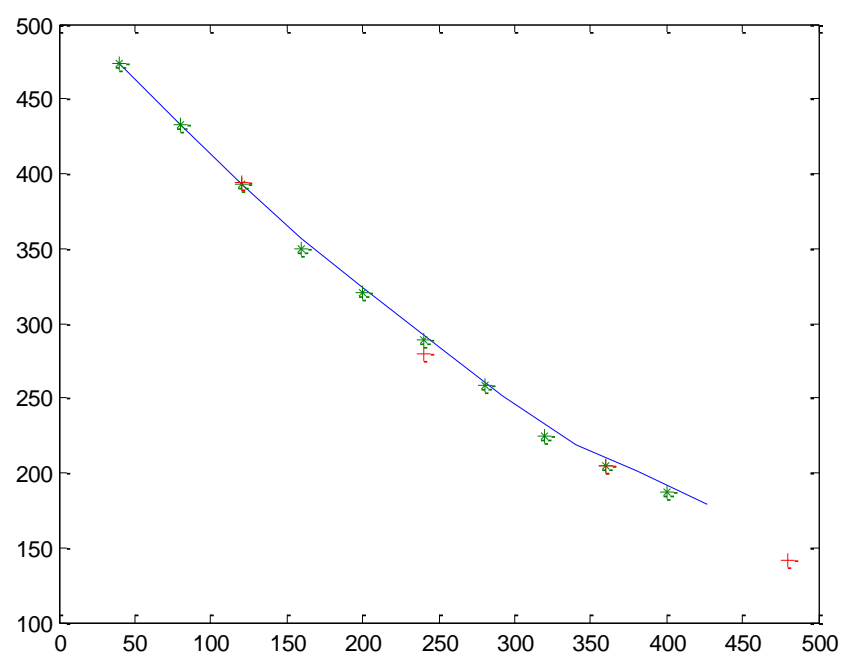

Fig. 3. Comparison - bi-level (-) and single optimization with $c=40 \mathrm{~s} \mathrm{(*)}$ and $c=120 \mathrm{~s} \mathrm{(+)}$

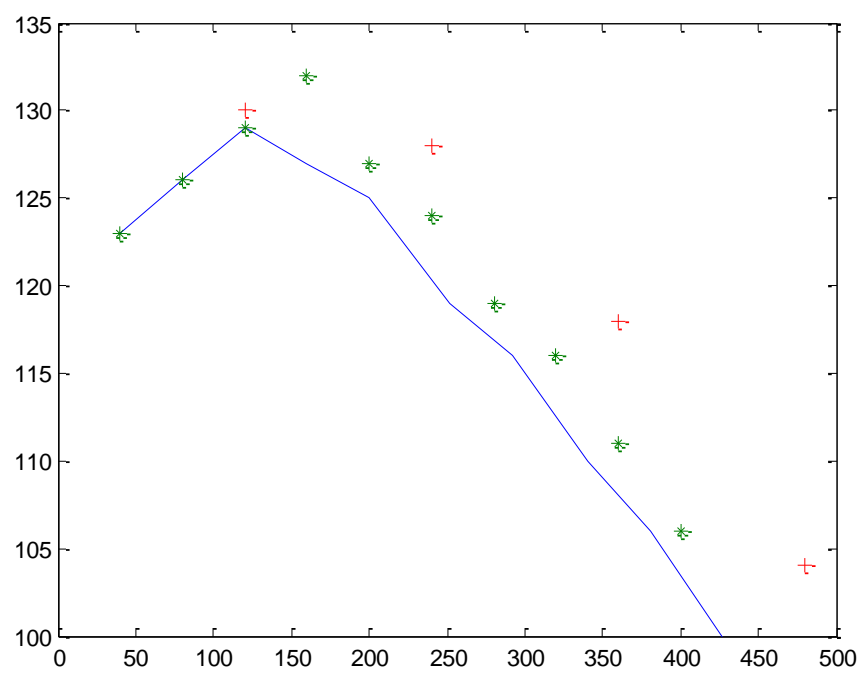

Fig. 4. Comparison bi-level (-) and single optimization $c=40 \mathrm{~s}(*)$ and $c=120 \mathrm{~s}(+)$

Fig. 3 presents the values of total waiting vehicles $x_{\text {total }}$ for a period of $500 \mathrm{~s}$ of simulation. The results are pretty the same for the three optimization problems. The graphics on Fig. 3 behave itself very close to each other.

On Fig. 4 the results are given for the waiting times for the priority directions. Following Fig. 4 it is seen that after a period of $150 \mathrm{~s}$ the bi-level control policy has advantages and provides less waiting time in comparison with the single optimization problems. 


\section{Conclusions}

This paper presents a formal bi-level optimization model for real time evaluation of the traffic lights duration and the traffic cycle. The bi-level approach gives advantages for the control policy and the management of the traffic flows in comparison with the classical optimization approaches. Hence, the new, more complicated formalism added value for practical problems related with traffic flows management. The results of the bi-level control have been compared with the cases of single optimization of the vehicles queues. The simulation results prove that the bi-level approach gives benefits satisfying additional goal, which improves the transport behavior. The bi-level optimization gives benefits and improves the optimization functionalities which take place in the Intelligent Transportation Systems. It performs integration of control influences which benefit the implementation of ITS control.

Acknowledgements: This work is partly supported by project № ДФНП-156/12.05.2016, Program for career development of young scientists, BAS.

\section{References}

1. A u e r, A., S. F e e s e, S. L o c k w o o d. History of Intelligent Transportation Systems. Report No FHWA-JPO-16-329. Department of Transportation Intelligent Transportation Systems. May 2016. https://ntl.bts.gov/lib/59000/59200/59263/download1.pdf

2. B r o t c o r n e, L., M. L a b b e, P. M a r c o t t e, G. S a v a r d. A Bi-Level Model for Toll Optimization on a Multi-Commodity Transportation Network - Transportation Science, Vol. 35, 2001, No 4, pp. 345-358.

3. C o $1 \mathrm{~s}$ o n, B., P. M a r c o t t e, G. S a v a r d. An Overview of Bi-Level Optimization.- In: Ann. Oper. Res, Vol. 153, Springer, 2007, pp. 235-256.

4. Co n s t a n t i n, I., M. F 1 o r i a n. Optimizing Frequencies in a Transit Network: A Nonlinear BiLevel Programming Approach. - International Transactions in Operational Research, Vol. 2, 1995, pp.149-164.

5. C ô t é, J.-P., P. M a r c o t t e, G. S a v a r d. A Bilevel Modeling Approach to Pricing and Fare Optimization in the Airline Industry. - Journal of Revenue and Pricing Management, Vol. 2, 2003, pp. 23-36.

6. D e m p e, S. Annotated Bibliography on Bi-Level Programming and Mathematical Programs with Equilibrium Constraints. - Optimization, Vol. 52, 2003, No 3, pp. 333-359.

7. F 1 o r i a n, M., Y. C h e n. A Coordinate Descent Method for the Bi-Level o-d Matrix Adjustment Problem. - International Transactions in Operational Research, Vol. 2, 1995, pp. 165-179.

8. H e a r n, D. W., M. V. R a m a n a. Solving Congestion Toll Pricing Models. - In: P. Marcotte, Ed. Equilibrium and Advanced Transportation Modelling, Dordrecht, Kluwer Academic, 1998, pp. 109-124.

9. H o, H. W., S. C. W o n g, Housing Allocation Problem in a Continuum Transportation System. Transportmetrica, Vol. 3, 2007, No 1, pp. 21-39.

10. J i m o h, F., T. M c C 1 u s k e y. Self-Management in Urban Traffic Control: An Automated Planning Perspective.- In: Frances M. T. Brazier, Omer F. Rana, John C. Strassner, Eds. Autonomic Road Transport Systems. Springer, 2016, pp. 29-46.

11. K a r a, B. Y., V. V e r t e r. Designing a Road Network for Hazardous Materials Transportation. Transportation Science, Vol. 38, 2004, pp. 188-196. 
12. K h e i r k h a h, A., N. H. R e z a, M. B. M a s u m e. A Bi-Level Network Interdiction Model for Solving the Hazmat Routing Problem. - Special Issue: Transportation in Supply Chain Management, Vol. 54, 2016, Issue 2, pp. 459-471.

http://dx.doi.org/10.1080/00207543.2015.1084061

13. K o t s i a 1 o s, A., A. P o o 1 e. Autonomic Systems Design for ITS Applications: Modelling and Route Guidance. - In: Frances M. T. Brazier, Omer F. Rana, John C. Strassner, Eds. Autonomic Road Transport Systems. Springer, 2016, pp. 131-146.

14. L a b b e, M., P. M a r c o t t e, G. S a v a r d. A Bi-Level Model of Taxation and its Application to Optimal Highway Pricing. - Management Science, Vol. 44, 1998, pp. 1608-1622.

15. L a r s s o n, T., M. P a t r i k s s o n. Side Constrained Traffic Equilibrium Models - Traffic Management through Link Tolls. - In: P. Marcotte, S. Nguyen, Eds. Equilibrium and Advanced Transportation Modelling. Dordrecht, Kluwer Academic, 1998, pp. 125-151.

16. M i g d a 1 a s, A. Bilevel Programming in Traffic Planning: Models, Methods and Challenge. Journal of Global Optimization, Vol. 7, 1995, No 4, pp. 381-405.

17. Q u r e s h i, N. K., A. H. A b d u 11 a h. A Survey on Intelligent Transportation Systems. - MiddleEast Journal of Scientific Research, Vol. 15, 2013, No 5, pp. 629-642.

18. S t o i 1 o v, T., K. S t o i 1 o v a, M. P a p a g e or g i o u, I. P a p a m i c h a i l. Bi-Level Optimization in a Transport Network. - Cybernetics and Information Technologies, Vol. 15, 2015, No 5, pp. 37-49.

19. S t o i 1 o v, T., K. S t o i 1 o v a, V. S t o i 1 o v a. Bi-Level Formalization of Urban Area Traffic Lights Control.- In: S. Margenov et al., Eds. Innovative Approaches and Solutions in Advanced Intelligent Systems. Vol. 648. Chapter 20. Springer, 2016, pp. 303-318.

20. S t o i 1 o v a, K., T. S t o i 1 o v, K. N i k o 1 o v. Autonomic Properties in Traffic Control. Cybernetics and Information Technologies, Vol. 13, 2013, No 4, pp. 18-32.

21. V i c e $\mathrm{n}$ t e, L. N., P. H. C a 1 a m a i. Bilevel and Multilevel Programming: A Bibliography Review. - Journal of Global Optimization, Vol. 5, 2004, No 3, pp. 291-306. 